\title{
Validation of state-space models with time-varying parameter uncertainty
}

\author{
Andreas Johansson
}

\begin{abstract}
The model validation problem assuming timevarying parameter uncertainty is addressed. A particular form of this uncertainty description, here named the biaffine input/output system is shown to be quite general and yet lead to simple solutions of the model validation problem, in the form of linear feasibility problems. An overhead crane is used as an illustrative example.
\end{abstract}

\section{INTRODUCTION}

Most of the contemporary approaches to feedback control require a description of the process, not only of its nominal behavior but also of the uncertainties associated with this description. How to achieve such a description, given a nominal model and a set of measurement data, is thus an active area of research known as model quality estimation. Tightly linked to this is the question of model validation, which, in the deterministic setting, is the question whether a given set of measurement data could have been produced by a given nominal model and uncertainty description.

Apart from its use in modelling for the purpose of feedback control, model validation also has applications in its own right. Validating a process model on-line tells us if the process has changed since it was modelled, which may be an indication that there is a malfunction. In other words, this is a formulation of the fault detection problem. If models of different faulty behavior of the process are available, then model validation may also be used as a tool for distinguishing which model fits the measurement data best, i.e. fault isolation. Finally, invalidation of the process model may also be taken as an indication that the controller should be redesigned, which highlights the connection to the field of controller supervision.

The model quality estimation problem has been considered from different viewpoints, both in a stochastic [1], [2] and a deterministic [3], [4], [5], [6] setting. In these references, uncertainty in both $\mathrm{H}_{\infty}$ and $\mathrm{L}_{1}$ is considered, but the underlying assumptions seems to be that the "true process", and consequently also the perturbation, is a linear time-invariant system of high order. The deterministic model validation problem has been considered in both $\mathrm{H}_{\infty}$ and $\mathrm{L}_{1}$, for linear time-invariant perturbations but also for timevarying perturbations, see e.g. [7], [8], [9], [10], [11].

Assuming time-varying perturbations allows for a quite general process description since nonlinear behavior can also then also be accounted for. However, model quality estimation for time-varying perturbations appears to be a daunting problem. Therefore, instead of using dynamic uncertainty

The author is with the Control Engineering Group, Luleå University of Technology, SE-971 87 Luleå, Sweden, Email: andreas.johansson@ltu.se, Phone: +46920 492334 elements we shall here describe the real process as a linear system with (in general unknown) time-varying parameters which then constitute the model uncertainty. As will be shown in the sequel, this will make it possible to solve the model validation approximately as a linear feasibility problem. Furthermore, model quality estimation is expected to be possible to formulate as a linear optimization problem but this is left for future research.

The chosen uncertainty description is quite general but it has its prime advantage when dealing with physically motivated grey-box models, where the number of time-varying parameters can be expected to be small. Such models can take many forms, ranging from the first-order system with delay used for Ziegler-Nichols tuning of a PID-controller to the large-scale model obtained by reducing a distributed parameter model for e.g. a blast furnace or a paper pulp digester [12].

Of course, the prime drawback of assuming a parametric, time-varying uncertainty description is its possible inadequacy for describing unmodelled dynamics. However, if the process physics is reasonably well known, then the unmodelled dynamics can be expected to be confined to high frequencies and can then often be described with a parametric model (such as a time-delay or a fast pole).

As process description, a state-space system with affine dependence on the uncertain time-varying parameters is assumed. This structure is used frequently in robust control and estimation [13], [14] although then often in continuous time and with uncertainty in the output also. Using the Kronecker product $\otimes$, it can be expressed concisely as [15]

$$
\begin{aligned}
x(k+1)= & A x(k)+B u(k)+P(\pi(k) \otimes x(k)) \\
& +D \pi(k)+Q(\pi(k) \otimes u(k)) \\
y(k)= & C x(k)+\eta(k)
\end{aligned}
$$

where $A \in \mathrm{R}^{n \times n}, B \in \mathrm{R}^{n \times p}, D \in \mathrm{R}^{n \times m}, P \in \mathrm{R}^{n \times n m}$, $Q \in \mathrm{R}^{n \times m p}$, and $C \in \mathrm{R}^{q \times n}$ are constant matrices. The vector $x(k) \in \mathrm{R}^{n}$ represents the state and $u(k) \in \mathrm{R}^{p}$ is the measured input while $y(k) \in \mathrm{R}^{q}$ is the measured output and $\eta(k) \in \mathrm{R}^{q}$ is some additive disturbance. The vector $\pi(k) \in \mathrm{R}^{m}$ represents the parameter uncertainties, i.e. the deviation from the nominal parameter values. Note that they are allowed to enter both linearly and bilinearly with the state $x$ as well as with the input $u$, thus providing a fairly general description for how a small parameter deviation affects a linear process. In [15] it is shown how this structure is obtained, under some restrictions, from a 2nd order Taylor expansion of a general, nonlinear system with uncertain parameters. 
The output $y$ in (1) depends nonlinearly on the uncertainty $\pi$ which makes both model quality estimation and model validation a nontrivial problem. In this work we will therefore derive an approximation of the output which is affine in $\pi$ to make it possible to formulate a general model validation problem as a linear feasibility problem.

\section{A. Notation}

The identity matrix of order $n$ is denoted $I_{n}$ while $1_{n}$ is a vector of dimension $n$ with each element equal to one. For vectors, absolute value $|\cdot|$ and inequalities are to be interpreted element-wise. A useful property of the Kronecker product is

Property 1: Let $x \in \mathrm{R}^{n}$ and $y \in \mathrm{R}^{m}$ for arbitrary natural numbers $m, n$. Then $x \otimes y=\left(x \otimes I_{m}\right) y=\left(I_{n} \otimes y\right) x$

In this paper, we shall be concerned with discrete time signals, represented by one-sided sequences, i.e. functions $\mathrm{Z}^{+} \rightarrow \mathrm{R}^{n}$ where $\mathrm{Z}^{+}$denotes the nonnegative integers. The $l_{\infty}$-norm of a one-sided scalar-valued sequence $u$ is defined as $\|u\|_{\infty}=\sup _{k>0}|u(k)|$ and the corresponding signal space (i.e. the set of sequences that satisfies $\|u\|_{\infty}<\infty$ ) is denoted $l_{\infty}$. The space of vector valued signals of dimension $n$ where each element belongs to $l_{\infty}$ is denoted $l_{\infty}^{n}$. The truncation operator $\mathcal{T}_{\theta}$ is defined as

$$
\left(\mathcal{T}_{\theta} u\right)(k)= \begin{cases}u(k), & 0 \leq k \leq \theta \\ 0, & \text { otherwise }\end{cases}
$$

\section{THE BIAFFINE INPUT/OUTPUT SYSTEM}

In this section, we will derive an approximation of (1) whose output is affine in the the uncertainty.

Lemma 1: Consider the Taylor expansion of $y(k)$ in (1) with respect to $\left[\begin{array}{llll}\pi(0)^{T} & \pi(1)^{T} & \cdots & \pi(k-1)^{T}\end{array}\right]^{T}$ and let $\hat{y}(k)$ denote the constant and linear terms while $r(k)$ represent the terms of order two and higher. Then $\hat{y}(k)$ is generated by

$$
\begin{aligned}
\zeta(k+1)= & A \zeta(k)+B u(k) \\
\xi(k+1)= & A \xi(k)+P(\pi(k) \otimes \zeta(k))+D \pi(k)+ \\
& Q(\pi(k) \otimes u(k)) \\
\hat{y}(k)= & C(\zeta(k)+\xi(k))
\end{aligned}
$$

with initial state $\zeta(0)=x(0)$ and $\xi(0)=0$. By defining the disturbance $v=r+\eta$, the output of (1) may be expressed as

$$
y(k)=\hat{y}(k)+v(k)
$$

Remark 1: The term biaffine input/output system is due to the fact that the operator from the two inputs ( $\pi$ and $u$ ) to the output $y$ is affine in both inputs which will be clear from Lemma 2.

Proof. Using Property 1 and with the definitions

$$
\begin{aligned}
\tilde{A}(k) & =\left(A+P\left(\pi(k) \otimes I_{n}\right)\right) \\
f(k) & =B u(k)+\left(D+Q\left(I_{m} \otimes u(k)\right)\right) \pi(k) \\
& =B u(k)+\tilde{D}(k) \pi(k)
\end{aligned}
$$

(1) may be written as $x(k+1)=\tilde{A}(k) x(k)+f(k)$. By iteratively computing $x(1), x(2)$, et $c$. it is easy to see that the above has the closed form solution

$$
\begin{aligned}
x(k)= & \tilde{A}(k-1) \cdots \tilde{A}(0) x(0)+f(k-1)+ \\
& +\sum_{\jmath=0}^{k-2} \tilde{A}(k-1) \cdots \tilde{A}(\jmath+1) f(\jmath)
\end{aligned}
$$

so that

$$
\begin{aligned}
& x(k)=\left(\prod_{\imath=0}^{k-1}\left(A+P\left(\pi(k-1-\imath) \otimes I_{n}\right)\right)\right) x(0) \\
& +\sum_{\jmath=0}^{k-2}\left(\prod_{\imath=0}^{k-\jmath-2}\left(A+P\left(\pi(k-1-\imath) \otimes I_{n}\right)\right)\right) B u(\jmath) \\
& +\sum_{\jmath=0}^{k-2}\left(\prod_{\imath=0}^{k-\jmath-2}\left(A+P\left(\pi(k-1-\imath) \otimes I_{n}\right)\right)\right) \tilde{D}(\jmath) \pi(\jmath) \\
& +B u(k-1)+\tilde{D}(k-1) \pi(k-1)
\end{aligned}
$$

The first terms of the above, is a product of factors of the form $\left(A+P\left(\pi(\cdot) \otimes I_{n}\right)\right)$. Expanding this yields a sum of products where each factor is either $A$ or $P\left(\pi(\cdot) \otimes I_{n}\right)$. By keeping only the terms with zero or one factor of the latter kind, we obtain a first order Taylor expansion with respect to $\pi$ as

$$
\begin{aligned}
\prod_{\imath=0}^{k-1}(A & \left.+P\left(\pi(k-1-\imath) \otimes I_{n}\right)\right) \\
\approx & A^{k}+P\left(\pi(k-1) \otimes I_{n}\right) A^{k-1} \\
& +A P\left(\pi(k-2) \otimes I_{n}\right) A^{k-2}+\cdots \\
& +A^{k-2} P\left(\pi(1) \otimes I_{n}\right) A+A^{k-1} P\left(\pi(0) \otimes I_{n}\right) \\
= & A^{k}+\sum_{\imath=0}^{k-1} A^{\imath} P\left(\pi(k-1-\imath) \otimes I_{n}\right) A^{k-1-\imath}
\end{aligned}
$$

The first sum in (3) may be treated in the same manner while the second sum has a factor $\pi(\jmath)$ on each term, so that only the term $A^{k-\jmath-1}$ must be retained when expanding the product. In conclusion, the following expression for the first order Taylor expansion of $x(k)$ is obtained

$$
\begin{aligned}
\hat{x}(k) & =A^{k} x(0)+\sum_{\imath=0}^{k-1} A^{\imath} P\left(\pi(k-1-\imath) \otimes I_{n}\right) A^{k-1-\imath} x(0) \\
& +\sum_{\jmath=0}^{k-2} \sum_{\imath=0}^{k-\jmath-2} A^{\imath} P\left(\pi(k-1-\imath) \otimes I_{n}\right) A^{k-2-\jmath-\imath} B u(\jmath) \\
& +\sum_{\jmath=0}^{k-2} A^{k-\jmath-1} B u(\jmath)+\sum_{\jmath=0}^{k-2} A^{k-\jmath-1} \tilde{D}(\jmath) \pi(\jmath) \\
& +B u(k-1)+\tilde{D}(k-1) \pi(k-1)
\end{aligned}
$$


Exchanging order of the summations in the double sum makes it possible to combine it with the first sum, so that

$$
\begin{aligned}
\hat{x}(k)= & A^{k} x(0)+\sum_{\imath=0}^{k-2} A^{\imath} P\left(\pi(k-1-\imath) \otimes I_{n}\right) \times \\
& \times\left(A^{k-1-\imath} x(0)+\sum_{\jmath=0}^{k-2-\imath} A^{k-2-\jmath-\imath} B u(\jmath)\right) \\
& +A^{k-1} P\left(\pi(0) \otimes I_{n}\right) x(0) \\
& +\sum_{\jmath=0}^{k-1} A^{k-\jmath-1} B u(\jmath)+\sum_{\jmath=0}^{k-1} A^{k-\jmath-1} \tilde{D}(\jmath) \pi(\jmath)
\end{aligned}
$$

Defining $\zeta(k)=A^{k} x(0)+\sum_{\jmath=0}^{k-1} A^{k-\jmath-1} B u(\jmath)$ yields

$$
\begin{aligned}
\hat{x}(k)= & \zeta(k) \\
& +\sum_{\imath=0}^{k-2} A^{\imath} P\left(\pi(k-1-\imath) \otimes I_{n}\right) \zeta(k-1-\imath) \\
& +\sum_{\jmath=0}^{k-1} A^{k-\jmath-1} \tilde{D}(\jmath) \pi(\jmath)+A^{k-1} P\left(\pi(0) \otimes I_{n}\right) x(0)
\end{aligned}
$$

Noting that $\zeta(0)=x(0)$ so that the last term can be combined with the second term and substituting $k-1-\imath$ for $\jmath$ in the same term gives

$$
\begin{aligned}
\hat{x}(k)= & \zeta(k)+\sum_{\jmath=0}^{k-1} A^{k-1-\jmath} P\left(\pi(\jmath) \otimes I_{n}\right) \zeta(\jmath) \\
& +\sum_{\jmath=0}^{k-1} A^{k-\jmath-1} \tilde{D}(\jmath) \pi(\jmath)
\end{aligned}
$$

Combining the last two terms while using the definition of $\tilde{D}$ and Property 1 finally results in

$$
\begin{aligned}
& \hat{x}(k)=\zeta(k)+ \\
& \sum_{\jmath=0}^{k-1} A^{k-1-\jmath}(P(\pi(\jmath) \otimes \zeta(\jmath))+D \pi(\jmath)+Q(\pi(\jmath) \otimes u(\jmath)))
\end{aligned}
$$

Denoting the second term of the above as $\xi(k)$ gives the realization (2).

\section{BOUNDS FOR DISTURBANCES AND PERTURBATIONS}

The disturbance $v(k)$ in (2) consists of both a linearization error $r(k)$ and a noise term $\eta(k)$. To bound this disturbance we will use a discrete-time version of the window norm [15], [16]

$$
\|v\|_{w}=\sup _{t \geq 0} \int_{0}^{t} w(t-\tau)|v(\tau)| d \tau
$$

where $w$ is nonnegative and bounded by a decreasing exponential function. To avoid the extra complications of extending the definition to vector-valued signals we assume a scalar measurement signal $(q=1)$ from now on and define

Definition 1: A window sequence is a sequence $w \in$ $\mathrm{Z}^{+} \rightarrow \mathrm{R}^{+}$which is not identically zero and satisfies $w(k) \leq$ $c e^{-a k}$ for all $k \geq 0$ and some positive $c$ and $a$.
Given a window sequence $w$, the window norm for discrete time signals may then be defined as

$$
\|v\|_{w}=\sup _{k \geq 0} \sum_{\imath=0}^{k} w(k-\imath)|v(\imath)|
$$

Remark 2: It is quite straightforward to show that (5) satisfies all properties of a norm, but the proof is omitted here to save space.

The window norm may be thought of as a generalization of the $l_{\infty}$-norm, which is obtained by choosing $w$ as the unit pulse function. Compared to the $l_{\infty}$-norm that only considers the peak value of the signal, the window norm is expected to be less conservative. The reason for this is that the window sequence $w$ will average the signal over a suitable time interval, e.g. a pulse function with nonunit duration or a decaying exponential function. In fact, by letting $w$ approach a unit step function (which is not a window function), the window norm approaches the $l_{1}$-norm. Compared to the $l_{p}$ norms, however, the window norm has the advantage that persistent signals, such as a step function, is bounded. It is straightforward to show that the signal space defined by $\left\{v \mid\|v\|_{w}<\infty\right\}$ is equal to the $l_{\infty}$-space but this proof is also left out here. In continuous time, however, the signal space of the window norm is larger than the $\mathrm{L}_{\infty}$-space [16].

Given a window function $w$, a set of admissible disturbances may now be expressed as the ball

$$
\mathrm{V}_{w, \epsilon}=\left\{v \in l_{\infty} \mid\|v\|_{w} \leq \epsilon\right\}
$$

For the parameter perturbations we shall assume bounds in the $l_{\infty}$-norm. Given a $\delta \in \mathrm{R}^{m}$, the set of admissible perturbations is defined as

$$
\mathrm{P}_{\delta}=\left\{\pi \in l_{\infty}^{m}|| \pi(k) \mid \leq \delta \text { for all } k \in \mathrm{Z}^{+}\right\}
$$

\section{Model VALidation}

Definition 2: Consider a process modelled by (2) with $v \in$ $\mathrm{V}_{w, \epsilon}$ and $\pi \in \mathrm{P}_{\delta}$. Let $\bar{y}$ be the measured output sequence from the process when subject to the input sequence $u$ and the initial state $x(0)=0$. Then the process model is said to be invalidated at $N$ if there exists no $(v, \pi) \in \mathrm{V}_{w, \epsilon} \times \mathrm{P}_{\delta}$ so that $\mathcal{T}_{N} y=\mathcal{T}_{N} y_{m}$.

In this section, we will derive a model validation test for the biaffine input/output system (2). For this, it is convenient to express the affine mapping from $\pi$ to $\hat{y}$ explicitly with matrix operations. By collecting the signals into vectors as

$$
\begin{aligned}
\Pi_{k} & =\left[\begin{array}{llll}
\pi(0)^{T} & \pi(1)^{T} & \cdots & \pi(k-1)
\end{array}\right]^{T} \\
Y & =\left[\begin{array}{llll}
y(0)^{T} & y(1)^{T} & \cdots & y(N)^{T}
\end{array}\right]^{T} \\
V & =\left[\begin{array}{llll}
v(0)^{T} & v(1)^{T} & \cdots & v(N)^{T}
\end{array}\right]^{T}
\end{aligned}
$$

and $\Pi=\Pi_{N}$, the following lemma may be formulated. 


$$
\begin{aligned}
\Omega & =\left[\begin{array}{cccc}
0 & 0 & \cdots & 0 \\
C P\left(I_{m} \otimes \zeta(0)\right) & 0 & \cdots & 0 \\
C A P\left(I_{m} \otimes \zeta(0)\right) & C P\left(I_{m} \otimes \zeta(1)\right) & & 0 \\
\vdots & \vdots & \ddots & \vdots \\
C A^{N-2} P\left(I_{m} \otimes \zeta(0)\right) & C A^{N-3} P\left(I_{m} \otimes \zeta(1)\right) & \ddots & 0 \\
C A^{N-1} P\left(I_{m} \otimes \zeta(0)\right) & C A^{N-2} P\left(I_{m} \otimes \zeta(1)\right) & \cdots & C P\left(I_{m} \otimes \zeta(N-1)\right)
\end{array}\right] \\
\Phi & =\left[\begin{array}{cccc}
C Q\left(I_{m} \otimes u(0)\right) & 0 & \cdots & 0 \\
C A Q\left(I_{m} \otimes u(0)\right) & C Q\left(I_{m} \otimes u(1)\right) & \cdots & 0 \\
\vdots & \vdots & \ddots & \vdots \\
C A^{N-2} Q\left(I_{m} \otimes u(0)\right) & C A^{N-3} Q\left(I_{m} \otimes u(1)\right) & \ddots & 0 \\
C A^{N-1} Q\left(I_{m} \otimes u(0)\right) & C A^{N-2} Q\left(I_{m} \otimes u(1)\right) & \cdots & C Q\left(I_{m} \otimes u(N-1)\right)
\end{array}\right]
\end{aligned}
$$

Lemma 2: Let $\Omega$ and $\Phi$ be defined as in (6) and let

$$
\begin{aligned}
\Psi & =\left[\begin{array}{cccc}
0 & 0 & \cdots & 0 \\
C D & 0 & \cdots & 0 \\
C A D & C D & & 0 \\
\vdots & \vdots & \ddots & \vdots \\
C A^{N-2} D & C A^{N-3} D & \ddots & 0 \\
C A^{N-1} D & C A^{N-2} D & \cdots & C D
\end{array}\right] \\
\Upsilon & =\left[\begin{array}{c}
C \zeta(0) \\
C \zeta(1) \\
\vdots \\
C \zeta(N)
\end{array}\right]
\end{aligned}
$$

and $\Xi=\Omega+\Psi+\Phi$. Then $Y=\Upsilon+\Xi \Pi+V$.

Proof. Property 1 applied to (4) gives

$$
\begin{aligned}
& \hat{x}(k)=\zeta(k)+\sum_{\jmath=0}^{k-1} A^{k-1-\jmath} \\
& \times\left(P\left(I_{m} \otimes \zeta(\jmath)\right) \pi(\jmath)+D \pi(\jmath)+Q\left(I_{m} \otimes u(\jmath)\right) \pi(\jmath)\right) \\
& =\zeta(k)+\left[\begin{array}{llll}
A^{k-1} D & A^{k-2} D & \cdots & D
\end{array}\right] \Pi_{k} \\
& +\left[A^{k-1} P\left(I_{m} \otimes \zeta(0)\right) \quad A^{k-2} P\left(I_{m} \otimes \zeta(1)\right)\right. \\
& \left.\cdots \quad P\left(I_{m} \otimes \zeta(k-1)\right)\right] \Pi_{k} \\
& +\left[A^{k-1} Q\left(I_{m} \otimes u(0)\right) \quad A^{k-2} Q\left(I_{m} \otimes u(1)\right)\right. \\
& \left.\cdots \quad Q\left(I_{m} \otimes u(k-1)\right)\right] \Pi_{k}
\end{aligned}
$$

From this, it is clear that $Y=\Upsilon+(\Omega+\Psi+\Phi) \Pi+V$ and the Lemma follows.

Lemma 2 formulates the process model as a linear equality. It remains to translate the assumption on disturbances and perturbations into linear inequalities. For $\pi(k), k=0 . . N-1$, the assumption $\pi \in \mathrm{P}_{\delta}$ may be expressed as $|\Pi| \leq 1_{N} \otimes \delta$ which, in turn, is equivalent to the linear inequalities

$$
\begin{aligned}
\Pi & \leq 1_{N} \otimes \delta \\
-\Pi & \leq 1_{N} \otimes \delta
\end{aligned}
$$

Since we are only using measurements from $k=0 . . N$ we can take $v(k)$ to be 0 for $k>N$. Thus, to check the assumption $v \in \mathrm{V}_{w, \epsilon}$ we only need to require that $\rho(k) \leq \epsilon$ for each $k \geq 0$ where

$$
\rho(k)=\sum_{\imath=0}^{\min (k, N)} w(k-\imath)|v(\imath)|
$$

If we furthermore assume that $w(k)$ is nonincreasing for $k>$ 0 then, for any $\jmath>0$,

$$
\begin{aligned}
\rho(N+\jmath) & =\sum_{\imath=0}^{\min (N+\jmath, N)} w(N+\jmath-\imath)|v(\imath)| \\
& =\sum_{\imath=0}^{N} w(N+\jmath-\imath)|v(\imath)| \\
& \leq \sum_{\imath=0}^{N} w(N-\imath)|v(\imath)|=\rho(N)
\end{aligned}
$$

and consequently, we only need to check that $\rho(k) \leq \epsilon$ for $k=0 . . N$. To translate this into linear inequalities we will use the following reasoning from [17]. Consider splitting $v$ into two sequences $v^{+}(k) \geq 0$ and $v^{-}(k) \geq 0$ such that $v^{+}(k)-v^{-}(k)=v(k)$. It is clear that $v^{+}(\imath)+v^{-}(\imath) \geq|v(\imath)|$ with equality iff either $v^{+}(\imath)=0$ or $v^{-}(\imath)=0$. Thus,

$$
\rho(k) \leq \sum_{\imath=0}^{\min (k, N)} w(k-\imath)\left(v^{+}(\imath)+v^{-}(\imath)\right)
$$

with equality if, for each $\imath$, either $v^{+}(\imath)=0$ or $v^{-}(\imath)=0$. Thus, with the definitions

$$
\begin{aligned}
V^{+} & =\left[\begin{array}{llll}
v^{+}(0) & v^{+}(1) & \cdots & v^{+}(N)
\end{array}\right]^{T} \\
V^{-} & =\left[\begin{array}{llll}
v^{-}(0) & v^{-}(1) & \cdots & v^{-}(N)
\end{array}\right]^{T}
\end{aligned}
$$

and

$$
W=\left[\begin{array}{cccc}
w(0) & 0 & \cdots & 0 \\
w(1) & w(0) & & 0 \\
\vdots & \vdots & \ddots & \\
w(N) & w(N-1) & \cdots & w(0)
\end{array}\right]
$$

the condition $\|v\|_{w} \leq \epsilon$ may be formulated as

$$
W\left(V^{+}+V^{-}\right) \leq \epsilon 1_{N+1}
$$




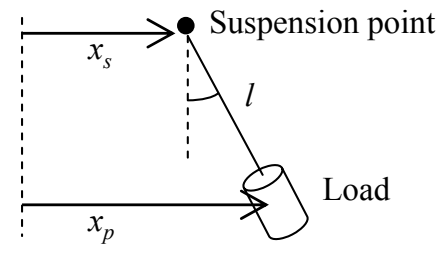

Fig. 1. The pendulum process

In conclusion, with $\chi^{T}=\left[\begin{array}{lll}\Pi^{T} & V^{+T} \quad V^{-T}\end{array}\right]$, the following theorem has been proved

Theorem 1: Consider a process modelled by (2) with a scalar output $y$ and $\pi \in \mathrm{P}_{\delta}$ and $v \in \mathrm{V}_{w, \epsilon}$ for a nonincreasing window sequence $w$. Let $\bar{y}$ be the measured output sequence from the process when subject to the input sequence $u$ and initial state $x(0)=0$ and define

$$
\bar{Y}=\left[\begin{array}{llll}
\bar{y}(0) & \bar{y}(1) & \cdots & \bar{y}(N)
\end{array}\right]^{T}
$$

Then the process model is invalidated at $N$ if the following linear feasibility problem has no solution.

$$
\begin{aligned}
& {\left[\Xi I_{N+1}-I_{N+1}\right] \chi=\bar{Y}-\Upsilon} \\
& {\left[\begin{array}{ccc}
I_{m N} & 0 & 0 \\
-I_{m N} & 0 & 0 \\
0 & W & W \\
0 & -I_{N+1} & 0 \\
0 & 0 & -I_{N+1}
\end{array}\right] \chi \leq\left[\begin{array}{c}
1_{N} \otimes \delta \\
1_{N} \otimes \delta \\
\epsilon 1_{N+1} \\
0 \\
0
\end{array}\right]}
\end{aligned}
$$

This feasibility problem has $2(N+1)+m N$ variables, $N+1$ equality constraints, and $3(N+1)+m N$ inequality constraints.

\section{Application to A PENDUlum Process}

As an illustration, we will apply the method to an overhead crane process with the position of the load $x_{p}$ as measurement $y_{c}$. The process is modelled as a pendulum (see Fig.1) where the horizontal velocity of the suspension point is proportional to the control signal $u_{c}$. With $x_{s}$ as the position of the suspension point, the linearized process may be formulated as

$$
\begin{aligned}
{\left[\begin{array}{c}
\dot{x}_{p} \\
\ddot{x}_{p} \\
\dot{x}_{s}
\end{array}\right] } & =\left[\begin{array}{ccc}
0 & 1 & 0 \\
-g / l & -c / m & g / l \\
0 & 0 & 0
\end{array}\right]\left[\begin{array}{l}
x_{p} \\
\dot{x}_{p} \\
x_{s}
\end{array}\right]+\left[\begin{array}{l}
0 \\
0 \\
b
\end{array}\right] u_{c} \\
y_{c} & =\left[\begin{array}{lll}
1 & 0 & 0
\end{array}\right]\left[\begin{array}{lll}
x_{p} & \dot{x}_{p} & x_{s}
\end{array}\right]^{T}+\eta_{c}
\end{aligned}
$$

where $g$ is the acceleration of gravity, $l$ is the length of the pendulum, $c$ is a friction coefficient, and $b$ is the proportionality factor converting the control signal into the speed of the suspension point.

The friction coefficient is not exactly known and will vary with the speed of the load and we assume that the proportionality factor $b$ is poorly known and possibly timevarying. The length of the pendulum $l$ will vary as the load is hoisted. We introduce the notations $\lambda=g / l$ and $\kappa=c / m$ which are thus functions of time.
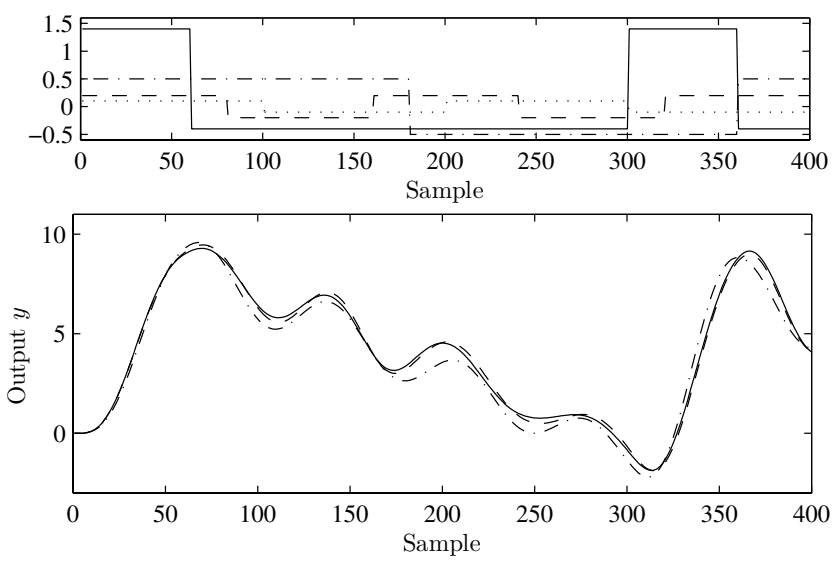

Fig. 2. Upper plot: Input $u(k)$ (solid), parameter perturbations $\pi_{1}(k)$ (dashed), $\pi_{2}(k)$ (dash-dotted), and $\pi_{3}(k)$ (dotted). Lower plot: Output of original system (dashed), biaffine approximation (solid), and nominal system (dash-dotted)

An Euler approximation gives the following discrete-time system

$$
\begin{aligned}
x(k+1) & =\left[\begin{array}{ccc}
1 & h & 0 \\
-h \lambda(k) & 1-h \kappa(k) & h \lambda(k) \\
0 & 0 & 1
\end{array}\right] x(k) \\
& +\left[\begin{array}{c}
0 \\
0 \\
h b(k)
\end{array}\right] u(k) \\
y(k) & =\left[\begin{array}{lll}
1 & 0 & 0
\end{array}\right] x(k)+\eta(k)
\end{aligned}
$$

where $u(k)=u_{c}(k h)$ and $y(k)=y_{c}(k h)$ while $\eta(k)$ is supposed to capture both the continuous-time noise term $\eta_{c}(k h)$ and the error introduced by the Euler approximation. The sampling interval in our simulations is $h=0.05$ seconds. Nominal values of $\lambda, \kappa$, and $b$ and time-varying deviations from these are introduced as

$$
\begin{aligned}
\kappa(k) & =\kappa_{0}+\pi_{1}(k) \\
\lambda(k) & =\lambda_{0}+\pi_{2}(k) \\
b(k) & =b_{0}+\pi_{3}(k)
\end{aligned}
$$

where $\kappa_{0}=0.25, \lambda_{0}=3.27$, and $b_{0}=2.0$. Thus, with the definitions

$$
\begin{aligned}
& A=\left[\begin{array}{ccc}
1 & h & 0 \\
-h \lambda_{0} & 1-h \kappa_{0} & h \lambda_{0} \\
0 & 0 & 1
\end{array}\right] \quad B=\left[\begin{array}{c}
0 \\
0 \\
h b_{0}
\end{array}\right] \\
& P=\left[\begin{array}{ccccccccc}
0 & 0 & 0 & 0 & 0 & 0 & 0 & 0 & 0 \\
0 & -h & 0 & -h & 0 & h & 0 & 0 & 0 \\
0 & 0 & 0 & 0 & 0 & 0 & 0 & 0 & 0
\end{array}\right] \\
& D=\left[\begin{array}{lll}
0 & 0 & 0 \\
0 & 0 & 0 \\
0 & 0 & 0
\end{array}\right] \quad Q=\left[\begin{array}{lll}
0 & 0 & 0 \\
0 & 0 & 0 \\
0 & 0 & h
\end{array}\right]
\end{aligned}
$$

the process may be expressed as (1).

To give some idea of the accuracy of the biaffine approximation (2), Fig. 2 (lower) shows a comparison between the 

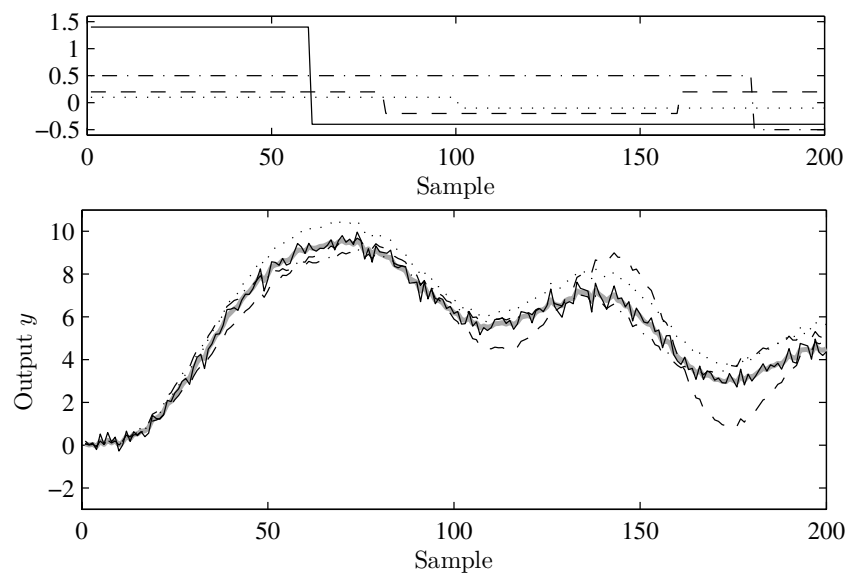

Fig. 3. Upper plot: Input $u(k)$ (solid), perturbations $\pi_{1}^{0}(k)$ (dashed), $\pi_{2}^{0}(k)$ (dash-dotted), and $\pi_{3}^{0}(k)$ (dotted). Lower plot: Output of Case 0 (thick,grey), Case 1 (dashed), Case 2 (dash-dotted), Case 3 (dotted), and Case 4 (solid, black). Case 1 to Case 4 are invalidated.

outputs of (1) and (2) with the parameter perturbations shown in the upper plot. Obviously, the biaffine input/output system is a significant improvement over the nominal system ((1) with $\pi$ identically zero).

To illustrate the sensitivity of the validation method, five test cases have been prepared. The parameter perturbations of the five cases are variations of the perturbations shown in Fig. 3, upper plot. The output of the original system (1) with an added white noise disturbance $\eta$ of standard deviation $\epsilon_{0}$ is chosen as measured output $\bar{y}$. The five test cases are

Case 0. $\pi=\left[\begin{array}{lll}\pi_{1}^{0} & \pi_{2}^{0} & \pi_{3}^{0}\end{array}\right]^{T}, \epsilon_{0}=0.1$
Case 1. $\pi=\left[\begin{array}{lll}3 \pi_{1}^{0} & \pi_{2}^{0} & \pi_{3}^{0}\end{array}\right]^{T}, \epsilon_{0}=0.1$
Case 2. $\pi=\left[\begin{array}{lll}\pi_{1}^{0} & 3 \pi_{2}^{0} & \pi_{3}^{0}\end{array}\right]^{T}, \epsilon_{0}=0.1$
Case 3. $\pi=\left[\begin{array}{lll}\pi_{1}^{0} & \pi_{2}^{0} & 3 \pi_{3}^{0}\end{array}\right]^{T}, \epsilon_{0}=0.1$
Case 4. $\pi=\left[\begin{array}{lll}\pi_{1}^{0} & \pi_{2}^{0} & \pi_{3}^{0}\end{array}\right]^{T}, \epsilon_{0}=0.3$

As bounds for the perturbations, the upper bounds of $\pi_{i}^{0}$ are chosen i.e. $\delta=\left[\begin{array}{lll}0.2 & 0.5 & 0.1\end{array}\right]^{T}$.

Since the process is oscillating, the disturbance $y-\hat{y}$ is dominated by the oscillation frequency. The window function $w$ in the norm $\|\cdot\|_{w}$ is thus chosen as a pulse function whose duration is one half oscillation period to provide averaging of the disturbance. This means

$$
w(k)= \begin{cases}1 / 35 & 0 \leq k<35 \\ 0 & \text { otherwise }\end{cases}
$$

To find a bound for the noise term $v$, Case 0 is simulated and the norm $\|y-\hat{y}\|_{w}$ is calculated and used as noise bound $\epsilon$. Thus, correct upper bounds are used for Case 0, and the model is consequently not invalidated for this case. In each of Case 1 to Case 3, one perturbation bound is exceeded by a factor three and in Case 4, the measurement noise is increased by a factor three. In all these cases the model is invalidated. The output $y$ for all cases are shown in Fig. 3, lower plot.

\section{CONCLUSIONS AND FUTURE WORK}

Time-varying parameters have been proposed as a setting for describing model uncertainty in linear systems. A particular form of this, here named the biaffine input/ouput system was shown to be a quite general uncertainty description and yet lead to simple solutions of the model validation problem, in the form of linear feasibility problems. An overhead crane was used as an illustrative example.

The next step is to develop methods for model quality estimation based on the biaffine input/output system. This can be formulated as a linear optimization problem, minimizing e.g. the size of the perturbations or disturbances or the variation in the perturbations, to name a few possibilities.

\section{ACKNOWLEDGMENTS}

The author wishes to thank the Hjalmar Lundbohm research center funded by LKAB for financing this research.

\section{REFERENCES}

[1] B. Ninness and G. C. Goodwin, "Estimation of model quality," Automatica, vol. 31, no. 12, pp. 1771-1797, 1995.

[2] W. Reinelt, A. Garulli, and L. Ljung, "Comparing different approaches to model error modeling in robust identification," Automatica, vol. 38, pp. 787-803, 2002.

[3] P. M. Mäkilä, J. R. Partington, and T. K. Gustafsson, "Worst-case control-relevant identification," Automatica, vol. 31, no. 12, pp. 17991819, 1995.

[4] L. Giarré, M. Milanese, and M. Taragna, " $H_{\infty}$ identification and model quality estimation," IEEE Transactions on Automatic Control, vol. 42, no. 2, pp. 188-199, 1997.

[5] M. Milanese and M. Taragna, " $H_{\infty}$ set membership identification: A survey," Automatica, vol. 41, pp. 2019-2032, 2005.

[6] V. F. Sokolov, "Control oriented model validation and errors quantification in the $\ell_{1}$ setup," IEEE Transactions on Automatic Control, vol. 50, no. 10, pp. 1501-1508, 2005.

[7] R. S. Smith and J. C. Doyle, "Model validation: A connection between robust control and identification," IEEE Transactions on Automatic Control, vol. 37, no. 7, pp. 942-952, 1992.

[8] K. Poolla, P. Khargonekar, A. Tikku, J. Krause, and K. Nagpal, "A time-domain approach to model validation," IEEE Transactions on Automatic Control, vol. 39, no. 5, pp. 951-959, 1994.

[9] J. Chen, "Frequency-domain tests for validation of linear fractional uncertainty models," IEEE Transactions on Automatic Control, vol. 42, no. 6, pp. 748-760, 1997.

[10] G. Dullerud and R. Smith, "A nonlinear functional approach to LFT model validation," Systems \& Control Letters, vol. 47, pp. 1-11, 2002.

[11] T. Zhou, "On the reproduction of closed-loop experimental data by an LFT perturbed model set," International Journal of Control, vol. 76, no. 6, pp. 581-590, 2003.

[12] L. Ding, T. Gustafsson, and A. Johansson, "Model parameter estimation of simplified linear models for a continuous paper pulp digester," Journal of Process Control, vol. 17, no. 2, pp. 115-127, 2007.

[13] D. Xie and G. Xu, "Computation of performance robustness bounds for control systems with parameter uncertainties: An LMI approach," IEEE Proc.-Control Theory Appl., vol. 152, no. 6, pp. 675-682, 2005.

[14] C. E. de Souza, K. A. Barbosa, and A. Trofino, "Robust filtering for linear systems with convex-bounded uncertain time-varying parameters," IEEE Transaction on Automatic Control, vol. 52, no. 6, pp. 1132-1138, 2007.

[15] A. Johansson, M. Bask, and T. Norlander, "Dynamic threshold generators for robust fault detection in linear systems with parameter uncertainty," Automatica, vol. 42, pp. 1095-1106, July 2006.

[16] A. Johansson, "Shift invariant signal norms for fault detection and control," Systems \& Control Letters, 2007. doi:10.1016/j.sysconle.2007.07.002.

[17] M. A. Dahleh and I. J. Diaz-Bobillo, Control of Uncertain Systems. Prentice Hall, 1995. 